\title{
Pilot Balloon Malfunction Caused by Endotracheal Tube Bite Blocker
}

\author{
Jacob R Adams DO, Justin Hoffman RRT, Joe Lavelle RRT, and Eduardo Mireles-Cabodevila MD
}

\section{Introduction}

A patient's bite is a frequent cause of endotracheal tube (ETT) obstruction, which affects the volume and pressure delivered by the ventilator. Bite-related ETT obstruction is a frequent cause of ventilator alarm activation, and can cause respiratory failure, negative-pressure pulmonary edema, and ETT tube/pilot tube damage. Clinicians formerly used objects (syringes, tongue depressors, gauze) or devices intended for other goals (ie, oropharyngeal airways or intermolar devices) to prevent the patient from biting the ETT, but those devices were often makeshift, not designed for prolonged use, and had risks such as accidental dislodgement, ulcers, aspiration, ischemia, and temporomandibular joint injury. Thus, commercially available, plastic bite blocks, which encase and protect the ETT, are now commonly used. These bite blocks have a low profile and some double as tube holders. These devices come in single sizes for adults or children. They are easy to use and thus are becoming ubiquitous. The placement of a bite block on the ETT is mainly left to clinician intuition, and, although instructions and policies may exist, bite-block placement and troubleshooting come from experience.

\section{Case Summary}

A 78-year-old, $152 \mathrm{~cm}$ tall woman was transferred to our ICU for evaluation of acute respiratory failure. At the outside hospital she was treated for community-acquired pneumonia, and was noted to have jaw stiffness and limited mouth-opening, causing inability to eat or drink. She

The authors are affiliated with the Respiratory Institute, Division of Pulmonary, Allergy, and Critical Care Medicine, Cleveland Clinic, Cleveland, Ohio.

The authors have disclosed no conflicts of interest.

Correspondence: Eduardo Mireles-Cabodevila MD, Respiratory Institute, Mail Code G62, Cleveland Clinic, 9500 Euclid Avenue, Cleveland OH 44195. E-mail: mirelee@ccf.org.

DOI: $10.4187 /$ respcare. 02474 developed worsening respiratory failure and hypoxemia and was electively intubated. The intubation was described as very difficult due to limited mouth opening, even with the use of neuromuscular blockers. Intubation was achieved with a $7.0 \mathrm{~mm}$, cuffed ETT (Mallinckrodt/Covidien, Mansfield, Massachusetts), fixed at $21 \mathrm{~cm}$ at the teeth. Her medical history was notable for hypertension, hyperlipidemia, and osteoporosis.

Her physical exam was relevant for limited mouth opening $(<2 \mathrm{~cm})$. Her neck mobility was limited in all directions. She was awake and interactive. Her lung exam demonstrated scant bilateral basilar crackles. Her heart rate was regular and rhythmic, and there was trace lower extremity edema. The ETT was easily compressed by the teeth, so a bite blocker (Universal Bite Block, B\&B Medical Technologies, Orangevale, California) was placed by the respiratory therapist, and secured with a fastener (Anchor Fast Oral Endotracheal Tube Fastener, Hollister, Libertyville, Illinois).

Ear, nose, and throat and dentistry consultation concluded that the limited mouth opening was due to severe temporomandibular joint disease. She was unable to open her mouth more than 2 finger-widths, voluntarily or under paralysis. After 4 days of therapy her respiratory status improved. She passed a spontaneous breathing trial and was awake and interactive. Our extubation checklist includes checking for cuff leak. ${ }^{1,2}$ She did not have any leak, as measured via ventilator volume or auscultation. Due to the absence of cuff leak and concern about the risk of difficult reintubation, the extubation was aborted. Endoscopic exam of the larynx was inconclusive, due to partial visualization of the laryngeal structures. She was given intravenous steroids (dexamethasone, $8 \mathrm{mg}$ every $8 \mathrm{~h}$ ) for presumed laryngeal edema.

The next day another cuff leak test again found no leak. As part of routine protocol, cuff pressures were measured, and all averaged $20 \mathrm{~cm} \mathrm{H}_{2} \mathrm{O}$. The team was discussing the possibility of a tracheostomy, given her difficult airway. The respiratory therapist, recalling similar events, decided to check the pilot balloon line, and discovered that the bite block had kinked the pilot balloon tubing at its junction on the ETT (Fig. 1); as a result we saw a completely deflated pilot balloon while the ETT cuff remained inflated (Fig. 2). 


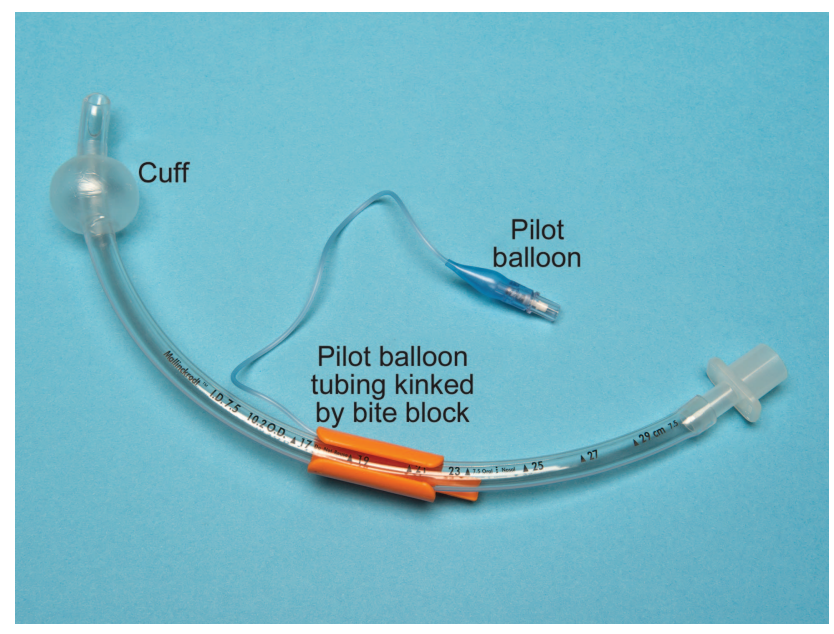

Fig. 1. Location at which the bite block typically kinks the pilot balloon tubing.

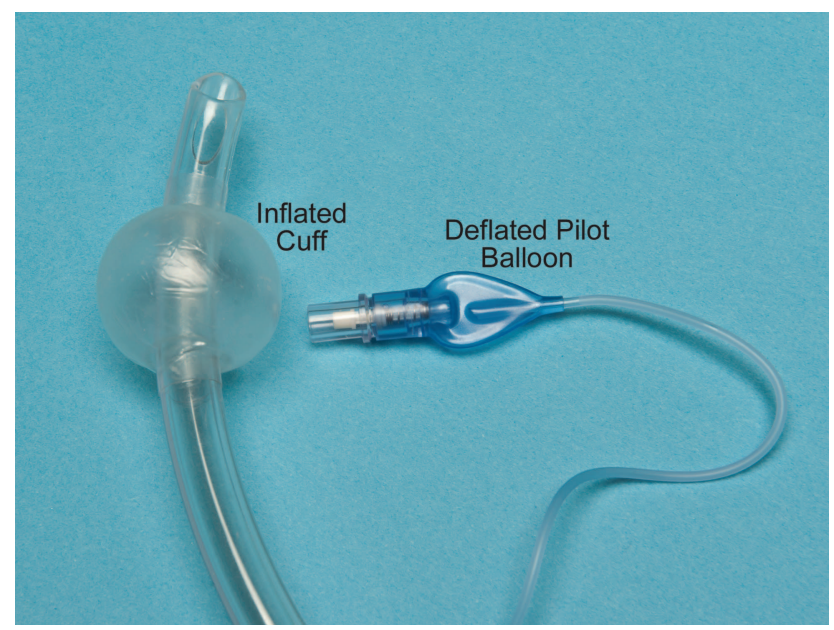

Fig. 2. When the pilot balloon tubing is kinked, as in Figure 1, the pilot balloon will be deflated while the cuff is completely inflated.

After removal of the bite block she passed another cuff leak test, and was then extubated over a tube exchanger. She continued to recover and was eventually discharged to the floor without further problems.

\section{Discussion}

Our case report highlights the need for specific steps with patients who have a bite block in place, because the bite block placement or its migration can kink the pilot balloon tubing, which, if not identified, can result in unnecessary interventions.

A kinked pilot tube can cause 2 scenarios:

- Persistent under-inflation of the cuff. The pilot balloon is fully inflated and the pressure gauge reading is normal, but the ETT cuff is deflated or underinflated. This could cause persistent air leak, inability to ventilate, and aspiration of fluids past the cuff. 3,4

- Persistent inflation or over-inflation of the cuff. The pilot balloon is deflated or fully inflated and the pressure gauge reading is normal, but the ETT cuff is inflated or overinflated. This could cause a false failure of the cuff leak test, inaccurate cuff pressure, and/or difficulty removing the ETT. ${ }^{5,6}$

Although we have experienced both scenarios, there is a paucity of reports referring to this problem. A systematic review of the literature revealed both situations scarcely being reported (Table 1). A stand-alone bite block that encases the ETT has been the most common device associated with pilot balloon occlusion. ${ }^{3-5}$ Each of the reported episodes had clinical consequences, which is likely why they reached publication. The reports by Alkire $^{3}$ and Brock-Utne ${ }^{4}$ describe large air leaks, desaturation, and difficulty ventilating the patient. In both cases, the pilot balloon was completely inflated while the ETT cuff was deflated. In a case similar to ours, Gleich ${ }^{5}$ et al and Singh et $\mathrm{al}^{6}$ report unintended kinking of the pilot tubing causing persistent inflation of the ETT cuff, which resulted in difficulty removing the ETT. Our extubation protocol includes a cuff leak test, and an absence of leak triggers an alert to the physician to decide whether or not the tube should be removed. Our patient had a difficult airway, so the decision was to provide steroids for $24 \mathrm{~h}^{7}$

Bite blocks can be grouped into those that serve only as bite blocks (which encase the ETT) and those that are part of an ETT holder. All the available bite blocks come in sizes from pediatric to adult. The cases reported in the literature have all involved bite blocks that were not part of an ETT holder. Those integrated with the ETT holder may have the advantage that they do not encase the ETT (or pilot balloon), and the bite blocker is not as long as the individual unit. Further, as they are attached to the patient's face, migration is less likely.

When we consider the usual depth of insertion of an ETT, the bite block length may go over the pilot balloon tubing take-off. The bite blocker we used was the adult size, which is $4.4 \mathrm{~cm}$ long. The location of the pilot balloon tubing take-off differs among the ETT sizes. Table 2 shows how the risk relates to ETT size, pilot balloon takeoff position, sex, and ETT length to the lips. The risk is higher in patients with short ETT insertion, distance to the lips, and larger ETT.

Each bite block comes with specific instructions on its placement. In this particular case, the bite block may be positioned to avert kinking the pilot balloon, but this does not allow the respiratory therapist to visualize the length of the tube at the patient's lip. Turning the bite block to visualize the numbering on the ETT will kink the pilot balloon when advancing the bite block (see Fig. 1). In- 


\section{Pilot Balloon Malfunction Caused by Endotracheal Tube Bite Blocker}

Table 1. Case Reports of Pilot Balloon Tubing Kinked by Endotracheal Tube Bite Block

\begin{tabular}{|c|c|c|c|c|c|c|}
\hline First Author & Year & $\begin{array}{l}\text { Model and/or } \\
\text { Manufacturer } \\
\text { of ETT }\end{array}$ & $\begin{array}{l}\text { ETT } \\
\text { Size } \\
\mathrm{mm}\end{array}$ & $\begin{array}{l}\text { Model and/or } \\
\text { Manufacturer } \\
\text { of Bite Block }\end{array}$ & Main Problem & Complications \\
\hline Alkire $^{3}$ & 1998 & Unknown & Unknown & $\begin{array}{l}\text { Bite Proof Bite Block, } \\
\text { B\&B Medical } \\
\text { Technologies }\end{array}$ & Tubing kinked by bite block & $\begin{array}{l}\text { Large air leak } \\
\text { Difficulty with oxygenation } \\
\text { and ventilation }\end{array}$ \\
\hline Brock-Utne ${ }^{4}$ & 2006 & Unknown & 8 & $\begin{array}{l}\text { Universal Bite Block, } \\
\text { B\&B Medical } \\
\text { Technologies }\end{array}$ & Tubing kinked by bite block & $\begin{array}{l}\text { Air leak } \\
\text { Desaturation }\end{array}$ \\
\hline Gleich $^{5}$ & 2008 & Hudson RCI & 9 & $\begin{array}{l}\text { B\&B Medical } \\
\text { Technologies }\end{array}$ & Tubing kinked by bite block & Difficulty removing ETT \\
\hline Singh $^{6}$ & 2009 & $\begin{array}{l}\text { The Laryngeal Tube, VBM } \\
\text { Medizintechnik }\end{array}$ & Unknown & Unknown & Tubing kinked by bite block & Difficulty removing ETT \\
\hline
\end{tabular}

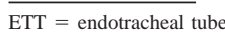

Table 2. Endotracheal Tube Sizes at Risk for Pilot Balloon Kinking by a Bite Blocker

\begin{tabular}{|c|c|c|c|}
\hline \multirow{2}{*}{$\begin{array}{l}\text { ETT } \\
\text { Size } \\
\text { mm }\end{array}$} & \multirow{2}{*}{$\begin{array}{c}\text { Pilot } \\
\text { Balloon } \\
\text { Take-Off } \\
\mathrm{cm}\end{array}$} & \multicolumn{2}{|c|}{ Risk Level* } \\
\hline & & $\begin{array}{c}\text { Male } \\
\text { (23 cm to lip) }\end{array}$ & $\begin{array}{c}\text { Female } \\
\text { (21 cm to lip) }\end{array}$ \\
\hline 6.0 & 15.0 & Low & Low \\
\hline 6.5 & 16.5 & Low & Low \\
\hline 7.0 & 18.0 & Low & Moderate \\
\hline 7.5 & 19.2 & Moderate & High \\
\hline 8.0 & 20.2 & High & Highest \\
\hline 8.5 & 21.2 & Highest & Highest \\
\hline 9.0 & 22.2 & Highest & Highest \\
\hline
\end{tabular}

* Based on a Mallinckrodt Lo-Pro cuffed endotracheal tube (ETT) and an adult-size Universal bite blocker.

deed, the manufacturer's instructions for the bite block we used $^{8}$ indicate that the pilot balloon should be placed between the bite block and the ETT. This may prevent the kinking at the pilot balloon take-off. The instructions read: "Should cuff filling problems occur, gently pull the pilot balloon line taut to remove any kinks." However, this would not solve the problem when the kinking is at the pilot balloon tubing take-off.

Whenever a pilot balloon is inflated, a clue to obstruction of the tubing is the amount of air needed to fill the ETT cuff. If a small volume is needed or the pressure reading rapidly rises while inflating, the clinician should suspect an obstruction of the pilot balloon line. When the balloon is deflated, a clue to an obstruction is a low volume removed with the syringe.

\section{Teaching Points}

- ETT bite blocks can obstruct the pilot balloon tubing.

- Every bite blocker has its particulars, but a larger ETT and a short ETT insertion distance to the lips are associated with a greater risk.

- Obstruction of the pilot balloon tubing can cause persistent inflation or under-inflation of the cuff.

- A clue that signals pilot balloon tubing obstruction is a small amounts of air (1-2 mL) being required to (apparently, not actually) deflate or inflate the cuff.

- Routine assessment of the intubated patient must include evaluation of the pilot balloon and tubing.

- We suggest that the ETT should be free of any additional device during extubation.

\section{REFERENCES}

1. Miller RL, Cole RP. Association between reduced cuff leak volume and postextubation stridor. Chest 1996;110(4):1035-1040.

2. Ochoa ME, Marín Mdel C, Frutos-Vivar F, Gordo F, Latour-Pérez J, Calvo E, Esteban A. Cuff-leak test for the diagnosis of upper airway obstruction in adults: a systematic review and meta-analysis. Intensive Care Med 2009;35(7):1171-1179.

3. Alkire MT. Ventilatory compromise secondary to occlusion of an endotracheal tube's balloon air channel by a malpositioned bite block. Anesthesiology 1998;88(5):1419.

4. Brock-Utne AJ. The universal bite-block: a word of caution. Anesth Analg 2006;103(2):495-496.

5. Gleich SJ, Mauermann WJ, Torres NE. An unusual cause of a difficult extubation. Respir Care 2008;53(3):376.

6. Singh M, Rautela RS, Kumar S. Laryngeal tube pilot balloon kinking in the presence of a bite block. Can J Anaesth 2009;56(11): 880-881.

7. McCaffrey J, Farrell C, Whiting P, Dan A, Bagshaw SM, Delaney AP. Corticosteroids to prevent extubation failure: a systematic review and meta-analysis. Intensive Care Med 2009;35(6):977-986.

8. B\&B Medical Technologies. B\&B Universal Bite Block protocol. 2008. http://bandb-medical.com/wp-content/uploads/2012/10/ 11160P1.pdf. Accessed November 27, 2013. 